$\mathrm{CO}_{2}$ must probably be stabilized at levels below 500 ppm. Economic cost-benefit calculations suggest that measures to mitigate $\mathrm{CO}_{2}$ emissions should be taken within the next few years if a 500 ppm threshold is not be exceeded.

\section{Final Remarks}

Inertia in the both the global socioeconomic system and the climate system are large. More than one hundred years have passed since Arrhenius pointed out that emitted $\mathrm{CO}_{2}$ accumulates in the atmosphere and leads to global warming. It takes years to decades to negotiate appropriate strategies, to implement policy measures, to develop new technologies, to replace existing high-carbon emitting technologies, and to adapt infrastructure.

Still larger inertia is found in the climate system. Part of the $\mathrm{CO}_{2}$ emitted today will remain airborne for many centuries; global temperature and sea level responses lag behind the radiative forcing imposed by greenhouse gases and other forcing agents as it takes many centuries to heat the water masses of the ocean. Still greater inertia is found when considering the response of the large ice sheets.

Since trends in society in general and in the global climate system cannot be changed immediately, in the final analysis one must say that if potentially adverse climate changes do indeed materialise then any trend may be a persistent one, or even become aggravated for some time.

According to Revelle and Suess:

"Human beings are now carrying out a large-scale geophysical experiment of a kind that could not have happened in the past nor be reproduced in the future. Within a few centuries we are returning to the atmosphere and oceans the concentrated organic carbon stored in sedimentary rocks over hundreds of millions of years."

\section{Acknowledgments}

C. Apenzeller and N. Gruber are thanked for their very thoughtfu comments, and Th. Stocker for continuous support.

\section{Further Information}

T. Boden et al., Trends '93: A Compendium of Data on Global Change (Carbon Dioxide Information Analysis Center) 1994.

J.T. Houghton et al., Climate Change 1994: Radiative Forcing of Climate Change and an evaluation of the IPCC IS92 emission scenarios. Report of working group I and III of the Intergovernmental Panel on Climate Change (IPCC, Cambridge University Press, UK) 1994.

J.T. Houghton et al., Climate Change 1995 -The Science of Climate Change: Contribution of WGI to the Second Assessment Report of the Intergovernmental Panel on Climate Change (IPCC, Cambridge University Press, UK) 1996.

U. Siegenthaler \& J.L. Sarmiento, Nature 365 (1993) 119.

\title{
A Hint of Deconfinement
}

\author{
Claudie Gerschel, Institut de Physique Nucléaire d'Orsay,CNRS-Université Paris 11, Orsay, and \\ Louis Kluberg, Ecole Polytechnique-CNRS, Palaiseau, report that a hint for the existence of the \\ elusive quark-gluon plasma needs confirmation.
}

The search for the phase transition of ordinary matter to the very peculiar quark-gluon plasma state (QGP for short) is a challenge for hundreds of physicists around the world. QGP is a conjectured very high density state of matter considered as the likely transient ancestor of usual nuclear matter. It is supposed to have filled the Universe at very early times, some microseconds after the Big-Bang.

QGP, like normal matter, is made of coloured quarks and gluons, the wellknown elementary constituents described by quantum chromodynamics (QCD) in the frame of the Standard Model. Nevertheless, as opposed to what happens in stable normal matter, quarks are so close to each other in this medium, that the average colour potential they are exposed to is screened out. As a consequence, they are no longer bound together as they usually are in mesons and baryons. In this socalled deconfined state of matter, quarks and gluons are able to freely move in the medium like electrons in a normal plasma.

Although the phase transition leading to a QGP is predicted theoretically by QCD calculations on the lattice to occur for matter under extreme temperature and energy density conditions, the final proof has necessarily to be provided by direct or indirect experimental evidence. Starting in 1986, medium- and high-energy ion beams became available at Brookhaven in the USA and at CERN. They made possible the search for QGP formation in ultrarelativistic nucleus-nucleus collisions where it was believed that the required extreme conditions could be reached at best.

The QGP search requires signatures allowing clear unambiguous conclusions. Although several signatures have been proposed up to now, their general weakness is that even if QGP is formed in a laboratory experiment, it is an extremely short-lived state with a lifetime of some $10^{-24} \mathrm{~s}$. It quickly turns back to normal matter through the inverse hadronization phase transition. Any QGP property to be looked for will therefore be detectable only if it keeps memory of the transient phase. It has to survive hadronization without being completely wiped out by strong interactions. In this sense, among the pro- posed signatures, thermal photons and lepton pairs as well as short-lived particles produced at the early stages of the collision, can be considered among the most sensitive ones [H. Satz, CERN-TH/96-172].

Ten years ago, in 1986, H. Satz and T. Matsui made the prediction that $J / \psi$ production would be suppressed if QGP was formed. The original argument was that the preresonant cí pair state, made of a charm quark and its antiquark, would be unable to become the bound $\mathrm{J} / \psi$ resonance within the QGP medium because of the screening of the colour potential. Some months later, experiment NA 38 started at CERN an extensive experimental programme studying charmonium production on different targets with incident protons, oxygen and sulphur ions, at 450 and $200 \mathrm{GeV}$ per nucleon. The apparatus was able to detect, with a special $18 \mathrm{~m}$ long spectrometer, the muon pairs produced by interactions of an incident beam on a fixed target. It measured the complete kinematics of the muon pair and was thus able to identify both the muon pairs in the mass continuum produced by the Drell-Yan mechanism and vector meson resonances as the $\varrho, \omega, J / \psi$ and $\psi^{\prime}$ promptly decaying into two muons of well-defined invariant mass. The detector was also able to measure, on an event-by-event basis, the trans-

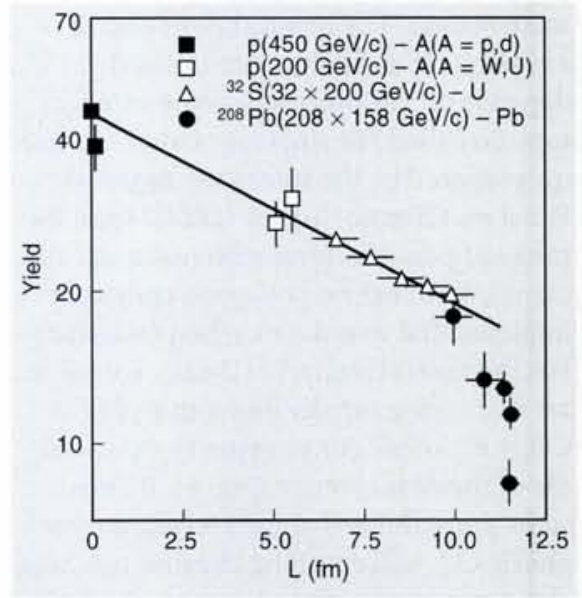

The yield of $\mathrm{J} / \psi$ as a function of the length $L$ of the path of the preresonant state through nuclear matter. DrellYan events, i.e., promptly produced muon pairs in the mass continuum, are used as a reference since their yield exhibits the normal expected behaviour. The $\mathrm{Pb}$ data are plotted in 5 centrality bins. 
verse energy produced in the reaction which allows one to estimate the centrality, or equivalently the impact parameter, of each individual collision.

The results obtained for $\mathrm{J} / \psi$ and $\psi^{\prime}$ production led to a solid, systematic reference. They are fully compatible with a mechanism of absorption, within the nucleus, of a preresonant state of the cic pair. This preresonant state is, by the way, of similar nature for the $\mathrm{J} / \psi$ resonance and its excited $\psi^{\prime}$ state. In the case of protoninduced reactions, this is the only mechanism actually needed to explain experimental observations. For this situation, moreover, since absorption is identical for $\mathrm{J} / \psi$ and $\psi^{\prime}$, the ratio of their production cross-sections is expected to be independent of the target nuclear size or, equivalently, of the atomic mass number $A$; this is the behaviour exhibited by the available experimental data. In addition, from the dependence of this $\mathrm{J} / \psi$ survival rate as a function of $A$, the value of the absorption cross-section can be calculated and amounts to 6-7 mb. In the case of ioninduced reactions up to sulphur, the same single mechanism with the same crosssection accounts for the experimental results on $\mathrm{J} / \psi$ production. Nevertheless, the suppression of the less bound $\psi^{\prime}$ state is significantly higher and the difference is attributed to destructive interactions with "comovers", the secondary particles produced in the reaction. According to theoretical considerations, comovers are nevertheless unable to significantly destroy the strongly bound $\mathrm{J} / \psi$ resonance, in the kinematical conditions of the experiment. If this is true, the absorption cross-section is expected to be the same for proton and sulphur-induced reactions, which is indeed in agreement with the data. Furthermore, in the case of proton-induced reactions, the overall comovers effect on $\psi^{\prime}$ should be extremely small in order to agree with the apparently constant $\psi^{\prime}$ to $\mathrm{J} / \psi$ production ratio.

With the acceleration of lead ions in 1994, CERN made available to experiments really heavy ion beams of $158 \mathrm{GeV}$ per nucleon. Conditions to form a QGP were better than with lighter ions like sulphur because larger volumes and larger number of participants are involved. These features favour thermalization of the medium and increase the lifetime of an eventual unstable QGP. Moreover, energy density significantly increases, at least locally, in the central part of the interaction volume. Experiment $\mathrm{NA}_{3} 8$ was upgraded to cope with the high radiation levels and multi- plicities induced by $\mathrm{Pb}-\mathrm{Pb}$ reactions. The detector of this new experiment, renamed NA50, was carefully tuned and fully understood in a run in 1994. During the following run, in the autumn of 1995, it took a significant amount of data including some 60000 events with a $J / \psi$ resonance, identified by the invariant mass of its two decay muons. The first preliminary results were reported in March 1996 at the Rencontres de Moriond and a more elaborate presentation was made at the end of May, at the Quark Matter'96 conference in Heidelberg.

The results show first of all that DrellYan events are produced according to their normal expected rate from proton-proton up to $\mathrm{Pb}-\mathrm{Pb}$ reactions. On the other hand, the production of the $\mathrm{J} / \psi$ resonance in $\mathrm{Pb}$ $\mathrm{Pb}$ reactions differs significantly from what could be anticipated from previous results obtained with protons and lighter ions. The production rate clearly deviates from the trivial extrapolation of nuclear absorption which is sufficient to account for previous observations (see figure).

The total yield of $J / \psi$ events amounts to $71 \pm 3 \%$ of what could be expected from past knowledge and the simple interpretation based on absorption. Moreover, as a function of the centrality of the reaction, the deviation from absorption increases with decreasing impact parameter. While the yield agrees, within errors, with classical expectations for the most peripheral reactions, it amounts to only $52 \pm 5 \%$ of the expected value for the most central collisions.

The safest conclusion that can be drawn is that nuclear absorption alone, as quantified with protons and lighter ions, cannot agree with the most recent $\mathrm{Pb}$ data. Something else has to be added. The most natural way to explain these observations is based on the assumption that a QGP has been formed. As an extreme scenario, it can be assumed that QGP is produced for energy densities immediately higher than those reached in sulphur-uranium reac-

\section{Italy Joins the ILL}

An agreement was signed on 29 October in Rome between INFM, the Italian Institute for the Physics of Matter, and the Institut Max Von Laue - Paul Langevin (ILL) in Grenoble which opens to the Italian community scientific partnership in the ILL for the next two years.

The agreement, signed by the INFM President, Professor C. Calandra and the ILL Director, Dr. R. Scherm, will give Italian scientists access to the world-class neutron research centre where about 800 experiments are currently carried out every year by some 1500 tions for which everything looks normal. Within this frame, it is possible to determine the $\mathrm{J} / \psi$ survival probability for $\mathrm{Pb}$ $\mathrm{Pb}$ reactions, taking into account the computed local energy density reached at each point of the interaction volume. The predicted suppression amounts to $66 \%$ of the pure absorption expectation - slightly below the measured value of $71 \pm 3 \%$, which could simply be the result of the extreme assumption.

Another way out of the problem calls upon destructive interactions of $\mathrm{J} / \psi$ with comovers. Although this mechanism is unnecessary to explain proton and sulphur-induced reactions, it has been artificially introduced in order to try to give a global description of all collisions up to $\mathrm{Pb}-\mathrm{Pb}$. While it is then possible to reproduce the strong absorption observed in $\mathrm{Pb}-\mathrm{Pb}$ interactions, the detailed agreement with all the experimental data is poor. The model fails to reproduce both the continuous behaviour between proton and sulphur-induced reactions and the apparent discontinuity observed with the $\mathrm{Pb}$-induced collisions.

Although the observations made by experiment NA50 seem to agree with what could be expected from a phase transition to QGP, it is difficult, with our present theoretical understanding, to account for the experimental results on both $\mathrm{J} / \psi$ and $\psi^{\prime}$ production for all proton and ioninduced reactions studied so far. In order to further constrain the models, more precise measurements are already on their way with incident protons and $\mathrm{Pb}$ ions and a possible future measurement could be considered with an intermediate ion like Sn. They will, hopefully, help to clarify the origin of the experimental observations and thereby confirm, or not, what could be considered today as a hint for QGP formation [For a review, see J.-P. Blaizot and J.Y. Ollitrault, in Quark-Gluon Plasma, Ed: R.C. Hwa ( World Scientific, Singapore, 1990) p.631].

guest scientists coming mostly from the member countries in Europe.

There has in the past been a fruitful collaboration between Italian scientists and ILL. So the scientific membership of INFM in ILL is an event long awaited by the Italian community, which now joins the ILL's other scientific members (Spain, Switzerland, Austria, and Russia) and the principal associated countries (France, Germany and the UK) in the scientific use and planning of the Institute.

Russian scientists gained the right earlier this year to join in research at the ILL following the completion of an agreement to supply $55 \mathrm{~kg}$ p.a. of highly enriched for nine years. 